\title{
Predictive Modelling of Human Walking Over a Complete Gait Cycle
}

\author{
Lei Ren $^{1,2}$, Richard K Jones ${ }^{1,3}$ and David Howard ${ }^{1,2}$ \\ ${ }^{1}$ Centre for Rehabilitation and Human Performance Research \\ ${ }^{2}$ School of Computing, Science and Engineering \& ${ }^{3}$ School of Healthcare Professions \\ University of Salford
}

Corresponding Author:

Prof. David Howard

School of Computing, Science and Engineering

University of Salford

Salford

M5 4WT

United Kingdom

Tel. 01612953584

Fax. 01612955575

Email d.howard@salford.ac.uk

Keywords: gait prediction, inverse dynamics, optimisation, optimal motor task

Word count (introduction through discussion): 3000

Submitted as an Original Article

(Submitted to Journal of Biomechanics) 


\begin{abstract}
An inverse dynamics multi-segment model of the body was combined with optimisation techniques to simulate normal walking in the sagittal plane on level ground. Walking is formulated as an optimal motor task subject to multiple constraints with minimisation of mechanical energy expenditure over a complete gait cycle being the performance criterion. All segmental motions and ground reactions were predicted from only three simple gait descriptors (inputs): walking velocity, cycle period and double stance duration. Quantitative comparisons of the model predictions with gait measurements show that the model reproduced the significant characteristics of normal gait in the sagittal plane. The simulation results suggest that minimising energy expenditure is a primary control objective in normal walking. However, there is also some evidence for the existence of multiple concurrent performance objectives.
\end{abstract}

\title{
Nomenclature
}

$$
\begin{array}{ll}
x_{a n}, y_{a n} & \text { coordinates of ankle joint centre in global reference frame } \\
\ddot{x}_{a n}, \ddot{y}_{a n} & \text { linear accelerations of ankle joint centre in global reference frame } \\
\Delta x_{a n} & \text { relative displacement of ankle joint along } x \text {-axis in stance phase } \\
x_{a n}^{(h s)} & x \text { coordinate of ankle joint at heel strike } \\
\theta_{f t}, \omega_{f t}, \alpha_{f t} & \text { angular displacement, velocity and acceleration of foot segment }
\end{array}
$$




\begin{tabular}{|c|c|}
\hline$x_{i}, y_{i}$ & coordinates of the $\mathrm{i}^{\text {th }}$ joint centre in global reference frame \\
\hline$\ddot{x}_{i}, \ddot{y}_{i}$ & linear accelerations of the $i^{\text {th }}$ joint centre in global reference frame \\
\hline$l_{j}$ & length of $\mathrm{j}^{\text {th }}$ body segment \\
\hline$\theta_{j}, \omega_{j}, \alpha_{j}$ & angular displacement, velocity and acceleration of body segment \\
\hline$m_{i}$ & mass of $i^{\text {th }}$ segment \\
\hline$\vec{a}_{i}$ & translational acceleration vector for the $\mathrm{i}^{\text {th }}$ segment's mass centre \\
\hline$\vec{F}_{j i}$ & $\mathrm{j}^{\text {th }}$ resultant joint force acting on the $\mathrm{i}^{\text {th }}$ segment \\
\hline$\vec{F}_{e i}$ & resultant external force acting on the $\mathrm{i}^{\text {th }}$ segment \\
\hline$\vec{g}$ & gravitational vector \\
\hline$I_{i}$ & moment of inertia of the $i^{\text {th }}$ segment \\
\hline$\theta_{i}, \alpha_{i}$ & angular displacement and acceleration of body segment \\
\hline$M_{j i}$ & net muscle moment acting on the $\mathrm{i}^{\text {th }}$ segment at the $\mathrm{j}^{\text {th }}$ joint \\
\hline$M_{e i}$ & resultant external moment acting on the $\mathrm{i}^{\text {th }}$ segment \\
\hline$M_{k i}$ & moment of the resultant joint force at the $\mathrm{k}^{\text {th }}$ joint acting on the $\mathrm{i}^{\text {th }}$ \\
\hline & segment \\
\hline$\vec{F}_{g i}, M_{g i}$ & ground reaction force and moment acting on left or right foot \\
\hline$T_{i}$ & net muscle torque acting at $\mathrm{i}^{\text {th }}$ joint \\
\hline$a_{0}^{(i)}, a_{k}^{(i)}, b_{k}^{(i)}$ & coefficients in Fourier series representing $\mathrm{i}^{\text {th }}$ segment angle trajectory \\
\hline$\omega$ & walking frequency \\
\hline$T_{c}$ & walking cycle period \\
\hline$\omega_{p}^{(i)}, \omega_{d}^{(i)}$ & angular velocity of proximal and distal segment at $i^{\text {th }}$ joint \\
\hline$E_{m}$ & mechanical energy expenditure over a walking cycle \\
\hline
\end{tabular}



$V_{a}$
average walking velocity over a walking cycle
$L$
ankle joint displacement over a walking cycle
$\mu_{x}$ friction coefficient between the foot and the ground surface

\section{Introduction}

Although the biomechanics of walking is well understood (McMahon, 1984; Zajac et al., 2003a, 2003b), little is known about the neural control strategies involved. Much of the research has been empirical, and few have focused on gait simulation (Chow and Jacobson, 1971, Davy and Audu, 1987; Marshall et al., 1989; Yamaguchi, 1990; Koopman, 1995; Anderson and Pandy, 2001). In predictive gait simulation, optimisation techniques have often been employed, where muscle forces and movements are determined by minimising a cost function.

The most popular approach to gait prediction has been to combine optimisation with forward dynamics, probably because this coincides with the natural sequence of neuromuscular control (Zajac and Winters, 1990; Yamaguchi, 1990, Pandy, 2001). However, since the system differential equations must be numerically integrated, the forward dynamics method leads to very long simulation times. In addition, realistic initial guesses for all control inputs (e.g. muscle activations) and initial values for all state variables (e.g. joint angular positions and velocities) are required to ensure that reasonable gait patterns can be obtained (Pandy, 2001). This depends on the availability of measurement data (Marshall et al., 1989; Anderson and Pandy, 2001) and compromises the capability of this approach as a predictive modelling tool. 
In contrast, the inverse dynamics method is very efficient computationally as it does not require numerical integration of the system differential equations. In addition, initial values for optimisation parameters can be set without the need for measurement data and initial values for the state variables are unnecessary. When inverse dynamics is applied in gait prediction, simple mathematical functions are used to represent the trajectories of the generalized coordinates (Yen and Nagurka, 1987; Koopman, 1995), where the function coefficients are the optimisation variables.

Only a few gait predication studies have employed inverse dynamics and optimisation (Yen and Nagurka, 1987; Channon, 1992; Koopman, 1995; Chevallereau and Aoustin, 2001). Most of these have considered only the single support phase or assume an instantaneous double support phase (zero duration). In addition, the foot segment was often neglected or assumed to be flat on the floor during stance. Moreover, additional trajectory constraints were often imposed on the segmental motions to simplify the optimisation problem. For example, Yen and Nagurka (1987) modelled the human skeletal system as a five-segment linkage. However, the trajectories of the body segments were only predicted for the single stance phase, the trunk was assumed to be upright throughout the cycle, and the model was forced to move at a constant forward speed. Koopman (1995) employed an eight-segment three-dimensional model to simulate normal walking over the whole gait cycle. However, all of the motions at the hip, knee and ankle were constrained to follow measured data or set to zero, the aim being to predict the unmeasured trunk and pelvic rotations, which were represented by Fourier series. 
In this paper, we present a combined inverse dynamics and optimisation method to predict normal human walking. In contrast to previous studies, the model predicts a complete gait cycle, including a normal double support phase. The foot segment is allowed to rotate freely during stance, rather than remaining flat on the floor. In addition, no predefined or measured trajectory constraints are imposed on segmental motions. The gait motions and joint torques are predicted from only three simple gait descriptors, average walking speed, cycle period and double stance duration, which minimizes the requirements for experimental data.

\section{Methods}

The multi-segment model

The human body was modelled as a planar (sagittal plane) seven-segment system (Figure 1). The interaction between the foot and the floor was modelled as a rigid contact, where the contact point is determined by the shape of the foot's plantar surface and the foot orientation.

Referring to Figure 1, the segmental angles $\theta_{1}, \theta_{2}, \ldots, \theta_{7}$ were used to describe the orientation of each body segment with respect to the global reference frame. In the double support phase, these segmental angles are not all independent because the model becomes a closed loop mechanism. The torques $T_{1}, T_{2}, \ldots, T_{6}$ are the net muscle moments acting on each joint to drive the multi-segment model. 
Anthropometric data, including segment masses, centre of mass positions and moments of inertia, are based on the data of de Leva (1996), which were modified for the HAT segment.

\section{Kinematics}

In this study, the stance foot was modelled as a rigid body with a curved surface rolling on the ground without slipping (Figure 2), such that the foot kinematics during the stance phase are described by

$$
\left\{\begin{array}{l}
\Delta x_{a n}=f\left(\theta_{f t}\right) \\
y_{a n}=g\left(\theta_{f t}\right)
\end{array}\right.
$$

where $\Delta x_{a n}=x_{a n}-x_{a n}^{(h s)}$, where $x_{a n}$ is the current $x$ coordinate of the ankle joint, and $x_{a n}^{(h s)}$ is the $x$ coordinate of the ankle joint at heel strike.

Equations (1) were determined using kinematic data captured in the gait laboratory using a six camera Qualisys motion analysis system, where the ankle joint was considered to be the mid-point between lateral and medial malleolus (Ren et al, 2005). Figure 3 shows the output of the foot model when the roll over shape is described by a best fit third order Fourier series. The relative timings of heel-strike and toe-off were also based on measurement data.

Differentiating Equations (1) twice, the accelerations of the ankle joint centre are, 


$$
\left\{\begin{array}{l}
\ddot{x}_{a n}=\frac{d^{2} f}{d \theta_{f t}^{2}} \cdot \omega_{f t}^{2}+\frac{d f}{d \theta_{f t}} \cdot \alpha_{f t} \\
\ddot{y}_{a n}=\frac{d^{2} g}{d \theta_{f t}^{2}} \cdot \omega_{f t}{ }^{2}+\frac{d g}{d \theta_{f t}} \cdot \alpha_{f t}
\end{array}\right.
$$

During walking there is at least one foot in contact with the ground throughout the gait cycle. Thus, the positions of the other joint centres in the multi-segment model were derived from the location of the stance ankle joint.

$$
\left\{\begin{array}{l}
x_{i}=x_{a n}+\sum_{j=1}^{m}\left(I(j) \cdot l_{j} \cdot \cos \theta_{j}\right) \\
y_{i}=y_{a n}+\sum_{j=1}^{m}\left(I(j) \cdot l_{j} \cdot \sin \theta_{j}\right)
\end{array}\right.
$$

where $m$ is the number of segments in the chain connecting the stance ankle joint to the $\mathrm{i}^{\text {th }}$ joint and $I(j)$ is a sign function, which is equal to 1 when the segment belongs to the stance limb, or equal to -1 if the segment is in the contralateral limb.

Differentiating Equation (3) twice, the accelerations of the joint centres are,

$$
\left\{\begin{array}{l}
\ddot{x}_{i}=\ddot{x}_{a n}-\sum_{j=1}^{m}\left(I(j) \cdot l_{j} \cdot\left(\alpha_{j} \cdot \sin \theta_{j}+\omega_{j}^{2} \cdot \cos \theta_{j}\right)\right) \\
\ddot{y}_{i}=\ddot{y}_{a n}-\sum_{j=1}^{m}\left(I(j) \cdot l_{j} \cdot\left(\omega_{j}^{2} \cdot \sin \theta_{j}-\alpha_{j} \cdot \cos \theta_{j}\right)\right)
\end{array}\right.
$$

Thus, given the segment angles, Equations (1) to (4) were used to calculate the coordinates of the joint centres and their accelerations. Thereafter, the positions and 
accelerations of each body segment mass centre were derived using anthropometric data.

\section{Kinetics}

The inverse dynamics method was employed to calculate joint kinetics and mechanical energy expenditure during walking. Since, in predictive modelling, the ground reactions are initially unknown, the inverse dynamics method must be based only on segmental motions. This differs from the conventional implementation of inverse dynamics used in gait laboratory studies (Winter, 1990; Siegler and Liu, 1997), where the calculations start from the measured ground reactions.

The equations of motion of the $i^{t h}$ body segment can be written as follows,

$$
\left\{\begin{array}{l}
m_{i} \cdot \vec{a}_{i}=\sum_{j=1}^{n_{i}} \vec{F}_{j i}+\vec{F}_{e i}+m_{i} \cdot \vec{g} \\
I_{i} \cdot \alpha_{i}=\sum_{j=1}^{n_{i}} M_{j i}+M_{e i}+\sum_{k=1}^{n_{i}} M_{k i}
\end{array}\right.
$$

where the segment has $n_{i}$ joints connecting it to other segments.

By combining the equations of motion of all body segments, the sums of the external forces and moments can be derived. Since, during walking, the only external forces and moments acting on the human body, other than gravity, are the ground reactions, these expressions can be written as,

$$
\left\{\begin{array}{l}
\sum \vec{F}_{g i}=\sum_{i=1}^{n} m_{i} \cdot\left(\vec{a}_{i}-\vec{g}\right) \\
\sum M_{g i}=\sum_{i=1}^{n} I_{i} \cdot \alpha_{i}-\sum_{i=1}^{n} \sum_{k=1}^{n_{i}} M_{k i}
\end{array}\right.
$$


where $n$ is the number of body segments in the model.

Therefore, during the swing phase, the ground reaction force acting on the single supporting foot can be obtained directly from Equation (6a). However, in double support phase, the ground reaction forces and moment (COP) are indeterminate. In order to solve this problem, the linear transfer assumptions shown in Figure 4, and introduced in Ren et al, 2005, have been used to model the transfer of the ground reactions from one foot to the other during the double support phase. As Figure 4 shows, these linear transfer assumptions are in good agreement with published ground reaction measurements (Winter, 1990).

During gait simulation, firstly, the ground reaction forces on each foot are calculated from Equation (6a) and the linear transfer relationships. Starting from the supporting feet and working up, segment by segment, the resultant force at each joint is calculated using Equation (5a). Then, the ground reaction moments on each foot are obtained from Equation (6b) and the linear transfer relationship for the centres of pressure. Starting from the feet and working up segment by segment again, the net muscle moments at each joint are calculated using Equation (5b). A detailed description of this inverse dynamics calculation process has been given elsewhere (Ren et al, 2005).

Optimisation and the constraints associated with gait

It has been observed in experimental studies that people's self-selected walking speed normally corresponds to minimum metabolic energy expenditure (Ralston, 1976; 
Cavagna and Kaneko, 1977). Therefore, in this study, the optimisation problem was described as: find segment trajectories that achieve the specified gait parameters, whilst minimizing energy cost, and satisfying the constraints associated with a walking gait.

The segment trajectories were represented by a set of Fourier series,

$$
\theta_{i}=a_{0}^{(i)}+\sum_{k=1}^{n}\left(a_{k}^{(i)} \cdot \cos (k \cdot \omega t)+b_{k}^{(i)} \cdot \sin (k \cdot \omega t)\right)
$$

where $n$ is the order of the Fourier series and $\omega=2 \pi / T_{c}$ is the walking frequency, where $T_{c}$ is the period of the gait cycle. One of the advantages of using a set of Fourier series is that they provide a representation of the gait motions that is implicitly cyclic, avoiding the need to introduce explicit constraints.

Power spectrum analysis of reflective marker data, during normal walking, has shown that most of the signal power $(99.7 \%$ ) is contained in frequencies below $6 \mathrm{~Hz}$ (Winter, 1990). Therefore, a set of $5^{\text {th }}$ order Fourier series were employed to represent the segmental rotations, resulting in a total of 11 Fourier coefficients for each segment, which were used as the optimisation parameters.

In normal walking, bilateral symmetry can be assumed, that is, movements of the left limb mirror movements of the right limb with a half cycle phase difference. Thus, the number of DOF representing the 7-segment model is reduced to 4, resulting in 44 Fourier coefficients being used as optimisation parameters. However, it should be 
noted that doing this does not impose any symmetry constraint on trunk motion. In fact, the optimiser can choose whichever pattern of trunk motion is most energy efficient.

As suggested by experimental observations of walking energetics (Ralston, 1976; Cavagna and Kaneke, 1977; Inman et al, 1994), a minimal energy criterion was employed as the objective function. In particular, the total joint work over the gait cycle was minimised.

Task constraints, biomechanical constraints and environmental constraints were implemented in the optimisation scheme. The task constraints (input gait descriptors) were average walking velocity $V_{a}$, cycle period $T_{c}$, and double stance duration. The biomechanical constraints prevent joint hyperextensions or other unrealistic movements. The environmental constraints represent the rules of ground interaction during walking.

All of the above leads to the following mathematical definition of the optimisation problem. Minimise mechanical energy expenditure over a complete gait cycle, which is defined as follows,

$$
\text { Minimise } \quad E_{m}=\int_{0}^{T_{c}} \sum_{i=1}^{n}\left|T_{i} \cdot\left(\omega_{p}^{(i)}-\omega_{d}^{(i)}\right)\right| d t
$$

where $T_{i}$ is the net muscle moment at the $\mathrm{i}^{\text {th }}$ joint, $\omega_{p}^{(i)}$ and $\omega_{d}^{(i)}$ are the angular velocities of the proximal and distal segments respectively. The optimisation parameters are $a_{0}^{(i)}, a_{k}^{(i)}, b_{k}^{(i)}(i=1,2,3,4, k=1,2,3,4,5)$, which are the coefficients of the 
$5^{\text {th }}$ order Fourier series representing the rotations of trunk, thigh, shank and foot.

Furthermore, the optimisation is subject to the following constraints:

(1) Segment motion constraints:

$0 \leq \theta_{i}(t) \leq \pi, t \in\left[0, T_{c}\right](i=1,2,3,4)$

(2) Joint motion constraints:

$\theta_{\min }^{(1)} \leq \theta_{2}(t)-\theta_{3}(t) \leq \theta_{\max }^{(1)}, \theta_{\min }^{(2)} \leq \theta_{4}(t)-\theta_{3}(t) \leq \theta_{\max }^{(2)}, t \in\left[0, T_{c}\right]$

(3) Kinematic constraints:

$y_{\text {tip }}(t)>0$ for a swing foot and $y_{t i p}(t)=0$ for a stance foot, where $y_{\text {tip }}$ is the vertical position of the foot's lowest point.

(4) Kinetic constraints:

$F_{y}(t)>0$ and $-\mu_{x}<\frac{F_{x}(t)}{F_{y}(t)}<\mu_{x}$ for a stance foot, where $\mu_{x}$ is the friction coefficient between the foot and the ground surface

(5) Stride length constraint:

$x_{a n}\left(T_{c}\right)-x_{a n}(0)=V_{a} \cdot T_{c}$

For the purposes of calculating the energy cost from the inverse dynamics calculations, 200 discrete calculation points were used over the gait cycle. The constraints defined above, and the representation of the segmental rotations by a set of finite Fourier series, ensure that solutions for this optimisation problem are valid cyclic walking gaits. However, this does not guarantee that they will be realistic.

The optimisation scheme was implemented in MATLAB using a Sequential Quadratic Programming (SQP) algorithm (Gill et al., 1981) from the optimisation toolbox. The three input gait descriptors (average walking velocity $V_{a}=1.5 \mathrm{~m} / \mathrm{s}$, gait cycle period 
$T_{c}=1.0 \mathrm{~s}$ and double stance duration $=0.18 \mathrm{~s}$ ) were obtained from the gait measurement data of one male subject (age: 38years, weight: $101.7 \mathrm{~kg}$, height: $178 \mathrm{~cm}$ ). A detailed description of the experimental procedures has been given elsewhere (Ren et al, 2005). The initial values of the optimisation parameters (Fourier coefficients) were set such that the model stands upright and stationary. In other words, except for the constant offset terms $\left(a_{0}^{(i)}\right)$, all of the Fourier coefficients were set to zero. In order to avoid finding a single local minimum, different initial values were randomly selected. These all represented stationary postures close to the upright position, as these were found to have a very good chance of converging to a solution. Due to the highly non-linear nature of the gait model, there appeared to be many local minima.

\section{Results}

Although many optimisation solutions were found, based on the major features of the gait patterns, they appeared to fall into 4 distinct families of solutions, with only small differences between members of the same family. We believe that these four families represent just four local minima and that the small differences are related to the precision of the optimisation process and the sensitivity of the objective function close to the true minima. The four gait patterns (families) are illustrated in Figure 5. Each family of gait patterns is represented by the member with the lowest energy cost. The gait patterns in Figures 5(a), 5(b) and 5(c) differ from normal walking in certain respects, which results in higher mechanical energy expenditure. The solution with the lowest energy consumption (Figure 5(d)) also yields the most realistic gait pattern. This suggests that deviations from a normal gait pattern lead to increased energy cost, 
which provides further evidence that minimisation of energy consumption is a feature of normal walking.

The predicted torso and lower limb joint rotations for the minimum energy solution are depicted in Figure 6 and compared with gait measurement data. Over most of the gait cycle, the majority of the predicted motions are in good agreement with the measurement data. The largest differences occur in the trunk segment. Although the overall trend agrees with the gait measurements and the reported data in the literature (Inman et al., 1994), the amplitude of fluctuation is noticeably larger. This difference could be due to the arms and pelvis not being considered, which probably moderate the trunk's angular fluctuations during normal walking. Another notable discrepancy is thigh rotation, which is much lower than the measured data shortly after opposite heel strike, thereby resulting in an increased range of thigh rotation. This is probably because the model does not include pelvic transverse rotation, which increases stride length, and the model compensates by increasing the thigh's angular displacement to achieve the specified stride length.

In Figure 7, the predicted ground reaction forces are compared with force plate data. Although agreement is reasonable where trends are concerned, there are unexpected fluctuations in the predicted forces. This probably arises from model simplifications, for example, because rotations of the pelvis are neglected.

\section{Discussion}


In this study, all segmental motions and ground reactions were predicted from only three simple gait descriptors: average forward velocity, gait cycle period and double stance duration, which minimizes the requirements for measurement data. No prescribed motion patterns or measured trajectories were imposed on the model. This is in contrast to previous work using a forward dynamics approach to gait prediction, where the initial and final kinematic states where taken to be as measured and imposed as optimisation constraints (Anderson and Pandy, 2001).

The predicted motions agree well with the measurement data over most of the gait cycle. The agreement with measured ground reaction forces is reasonable, but there are unexpected fluctuations. Moreover, among the local minima found, the solutions with the lowest energy consumption produced the most realistic gait patterns. This implies that minimizing energy cost is a primary motor control objective in normal walking. This seems a reasonable inference for the lower limbs, since it has been found that the cyclic movement of the legs accounts for the majority of the energy cost of walking (Pierrynowski et al, 1980). This is supported by the fact that the predicted motions of the lower limbs showed better agreement with the measured data than those of the trunk segment.

The large predicted trunk motions are partly explained by the fact that the arms and pelvis are not modelled. However, it has been shown in experimental studies that head motion is smoother than that of the pelvis and the shoulder (Cappozzo et al., 1978; Cappozzo, 1981), which may be due to the requirement to protect the visual and vestibular systems from excessive mechanical disturbance. If so, minimisation of head excursions, rather than energy cost, may be the primary control criterion for trunk 
motions. This suggests that multiple performance objectives are employed in human walking (Marshall et al, 1989).

The differences between the model predictions and experimental data are probably a result of the limitations of the seven-segment model. Many of the discrepancies may be due to the model being limited to the sagittal plane and the fact that the pelvis and arms have been omitted. Pelvic transverse rotation increases stride length and decreases the angular thigh excursion. Moreover, pelvic tilt can help to decrease and smooth the trajectory of the body mass centre (Inman et al, 1994).

The use of inverse dynamics, instead of forward dynamics, has several advantages including its computational efficiency, which is very important for predictive models that are based on optimisation techniques. Since no numerical integration of the differential equations is involved, the execution time for each optimisation iteration is greatly reduced. For example, the prediction model proposed in this paper required only 20 minutes of CPU time to converge to a minimal energy solution (Intel Pentium 4, 3.2 GHz). Another advantage of inverse dynamics is simpler implementation of the kinematic and kinetic constraints associated with walking.

The authors plan to extend this work by creating a full three dimensional gait prediction model. In addition, some of the variables that are currently fixed (gait cycle duration, stride length etc) could be free to vary during optimisation, allowing further investigation of the velocity-stride length relationship during human walking. 


\section{Acknowledgements}

Funding for this work has been provided by the UK Ministry of Defence. The assistance and support of Will Tutton (MoD) has been particularly valuable. 


\section{References}

Anderson, F.C. and Pandy, M.G. (2001). Dynamic optimisation of human walking. ASME Journal of Biomechanical Engineering 123, 381-390.

Cappozzo, A. (1981). Analysis of the linear displacement of the head and trunk during walking at different speed. Journal of Biomechanics 14, 411-426.

Cappozzo, A., Figura, A., Leo, T. and Marchetti, M. (1978). Movements and mechanical energy changes in the upper part of the human body during walking. In: Asmussen, E. and Jorgensen, K. (eds.), Biomechanics VI-A. Baltimore, MD: University Park Press.

Cavagna, G.A. and Kaneko, M. (1977). Mechanical work and efficiency in level walking and running. Journal of Physiology 268, 467-481.

Channon, P.H., Hopkins, S.H. and Pham, D.T. (1992). Derivation of optimal walking motions for a bipedal walking robot. Robotica 10, 165-172.

Chevallereau, C. and Aoustin, Y. (2001). Optimal reference trajectories for walking and running of a biped robot. Robotica 19, 557-569.

Chow, C.K. and Jacobson, D.H. (1971). An optimal programming study of human gait. Mathematical Biosciences 10, 239-306.

Davy, D.T. and Audu, M.L. (1987). A dynamic optimisation technique for predicting muscle forces in the swing phase of gait. Journal of Biomechanics 20, 187-201. de Leva, P. (1996) Adjustments to Zatsiorsky-Seluyanov's segment inertia parameters. Journal of Biomechanics 29, 1223-1230.

Gill, P.E., Murray, W. and Wright M.H. (1981). Practical Optimization, Academic Press, London.

Inman, V.T., Ralston, H.J. and Todd, F. (1994). Human locomotion. In: Rose, J. and Gamble J.G. (Eds.), Human Walking. $2^{\text {nd }}$ Edition. Williams \& Wilkins, Baltimore. 
Koopman, B., Grootenboer, H.J. and de Jongh, H.J. (1995). An inverse dynamics model for the analysis, reconstruction and prediction of bipedal walking. Journal of Biomechanics 28, 1369-1376.

Marshall, R.N., Wood, G.A. and Jennings, L.S. (1989). Performance objectives in human movement: A review and application to the stance phase of normal walking. Human Movement Science 8, 571-594.

McMahon, T.A. (1984). Muscles, Reflexes, and Locomotion. Princeton University Press, Princeton, NJ.

Pandy, M. G. (2001). Computer modeling and simulation of human movement. Annual Review of Biomedical Engineering 3, 245-273.

Pierrynowski, M.R., Winter D.A. and Norman R.W. (1980). Transfer of mechanical energy within the total body and mechanical efficiency during treadmill walking. Ergonomics 23, 147-156.

Ralston, H.J. (1976). Energetics of human walking. In: R.M. Herman et al., (Eds.). Neural Control of Locomotion. Plenum Press, New York.

Ren L., Jones R. and Howard D. (2005). Dynamic analysis of load carriage biomechanics during human level walking. Journal of Biomechanics 38(4), 853-863. Siegler, S. and Liu, W. (1997). Inverse dynamics in human locomotion. In: Allard P. et al. (Eds.), Three-dimensional Analysis of Human Locomotion. John Wiley and Sons Ltd, Wiley, New York.

Winter, D. A. (1990). The Biomechanics and Motor Control of Human Movement (2nd Edition). John Wiley and Sons Ltd, Wiley, New York.

Yamaguchi, G.T. (1990). Performing whole-body simulations of gait with 3-D, dynamic musculoskeletal models. In: Winters, J.M. and Woo, S.L.-Y. (Eds.), Multiple 
Muscle Systems. Biomechanics and Movement Organization. Spring-Verlag, New York.

Yen, V. and Nagurka, M.L. (1987). Suboptimal trajectory planning of a five-link human locomotion model. In ASME Winter Annual Meeting, Biomechanics of Normal and Prosthetic Gait, Boston, MA, USA.

Zajac, F.E. and Winters, J.M. (1990). Modeling musculoskeletal movement systems: joint and body-segment dynamics, musculoskeletal actuation, and neuromuscular control. In: Winters, J.M. and Woo, S.L-Y. (Eds.), Multiple Muscle Systems. Biomechanics and Movement Organization. Spring-Verlag, New York. Zajac, F.E., Neptune R.R. and Kautz, S.A. (2003a). Biomechanics and muscle coordination of human walking. Part I: Introduction to concepts, power transfer, dynamics and simulations. Gait and Posture 16, 215-232. Zajac, F.E., Neptune R.R. and Kautz, S.A. (2003b). Biomechanics and muscle coordination of human walking. Part II: Lessons from dynamical simulations and clinical implications. Gait and Posture 17, 1-17. 


\section{Figures and Captions}

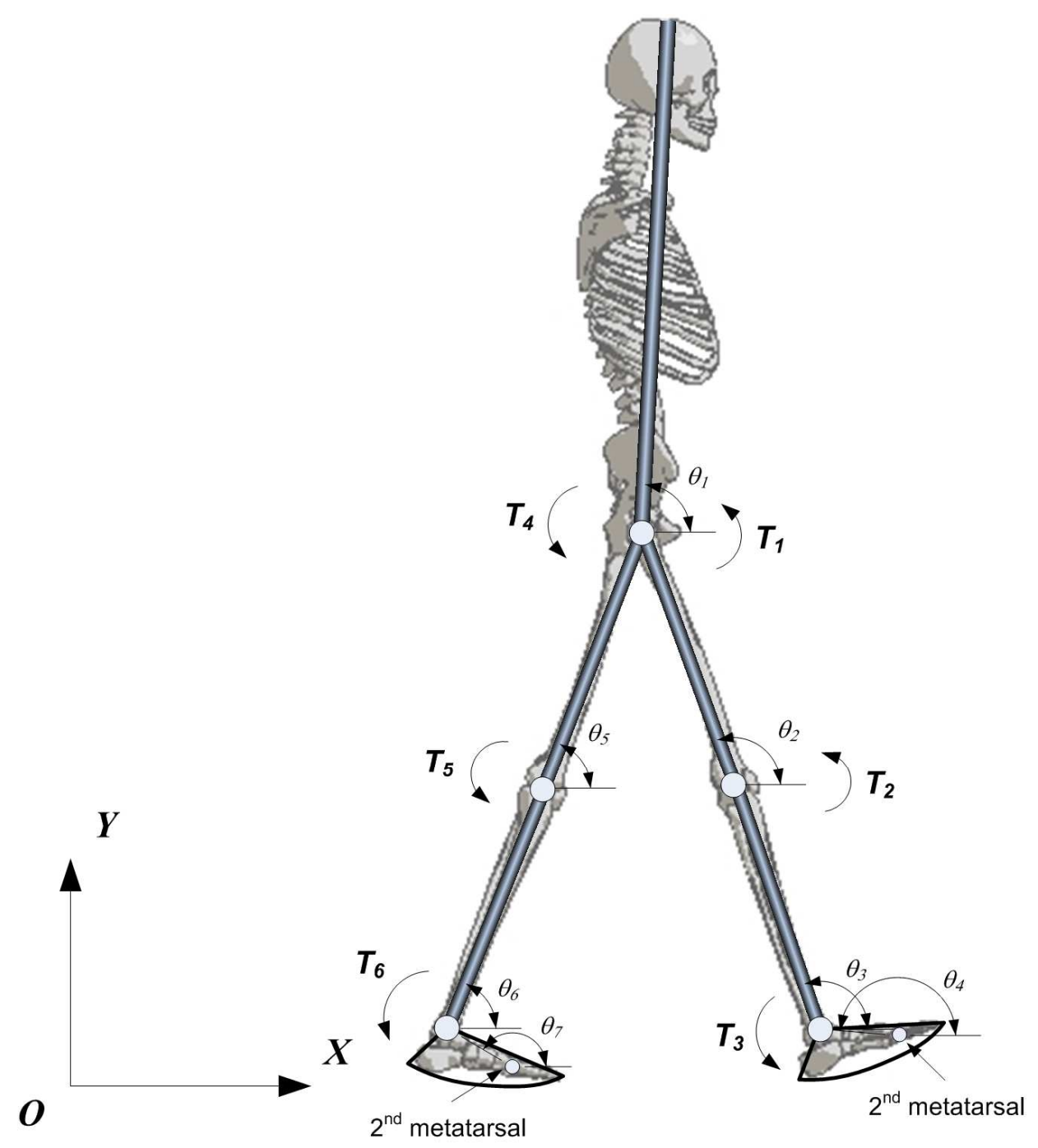

Figure 1 The seven-segment model including 6 joints and the following segments: the right and left thighs, shanks, and feet together with a HAT segment (head, arms and trunk). Segmental angles $\theta_{1}, \theta_{2}, \ldots, \theta_{7}$ are defined with respect to the X-axis of the global reference frame, counter-clockwise being positive. $T_{1}, T_{2}, \ldots, T_{6}$ are the net muscle moments acting at each joint, counter-clockwise being positive. 


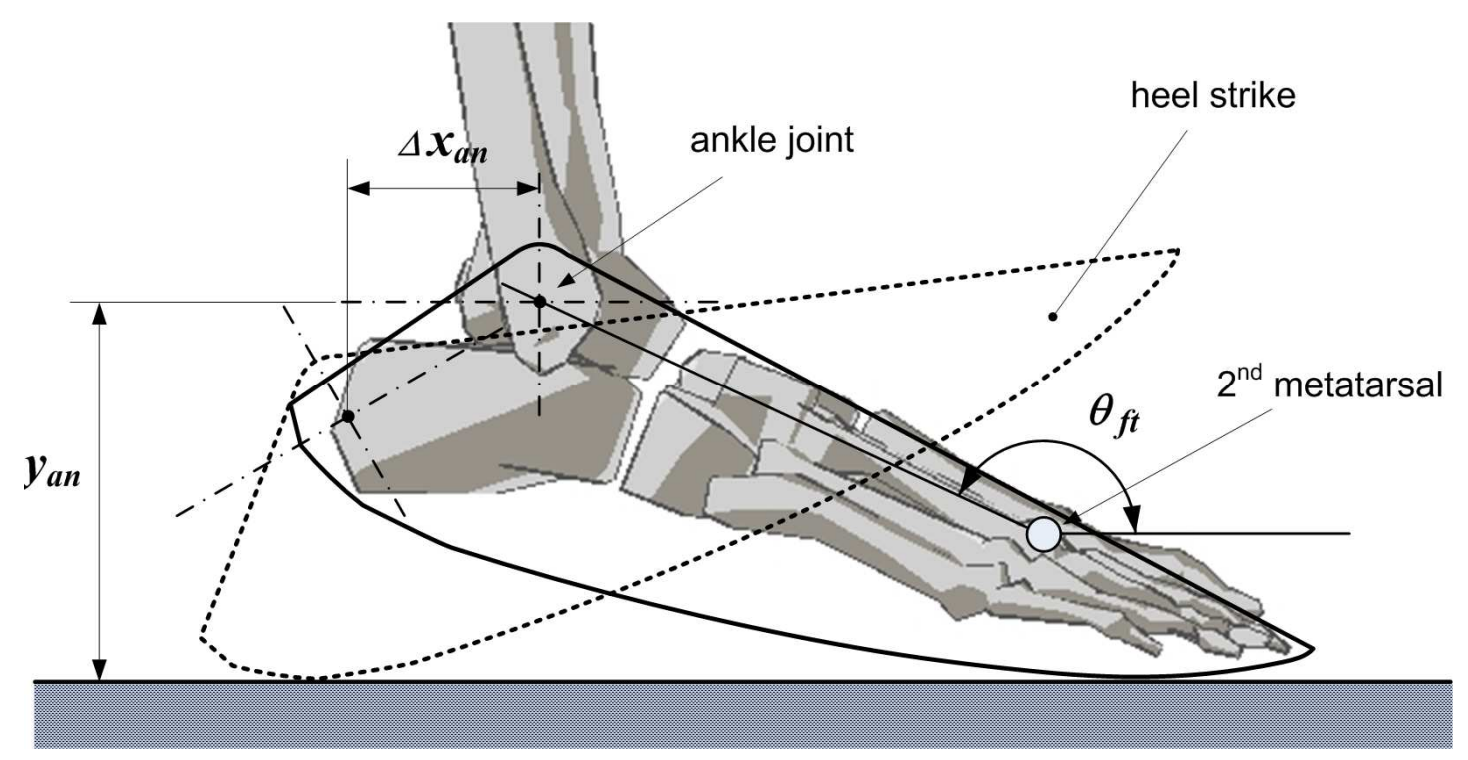

Figure 2 The ankle-foot kinematic relationships during foot rollover in the stance phase. The foot angular displacement is defined by the line connecting the ankle joint centre and the $2^{\text {nd }}$ metatarsal, and the $x$-axis. The displacement of the ankle joint along the $x$-axis is measured from the position at heel strike. 


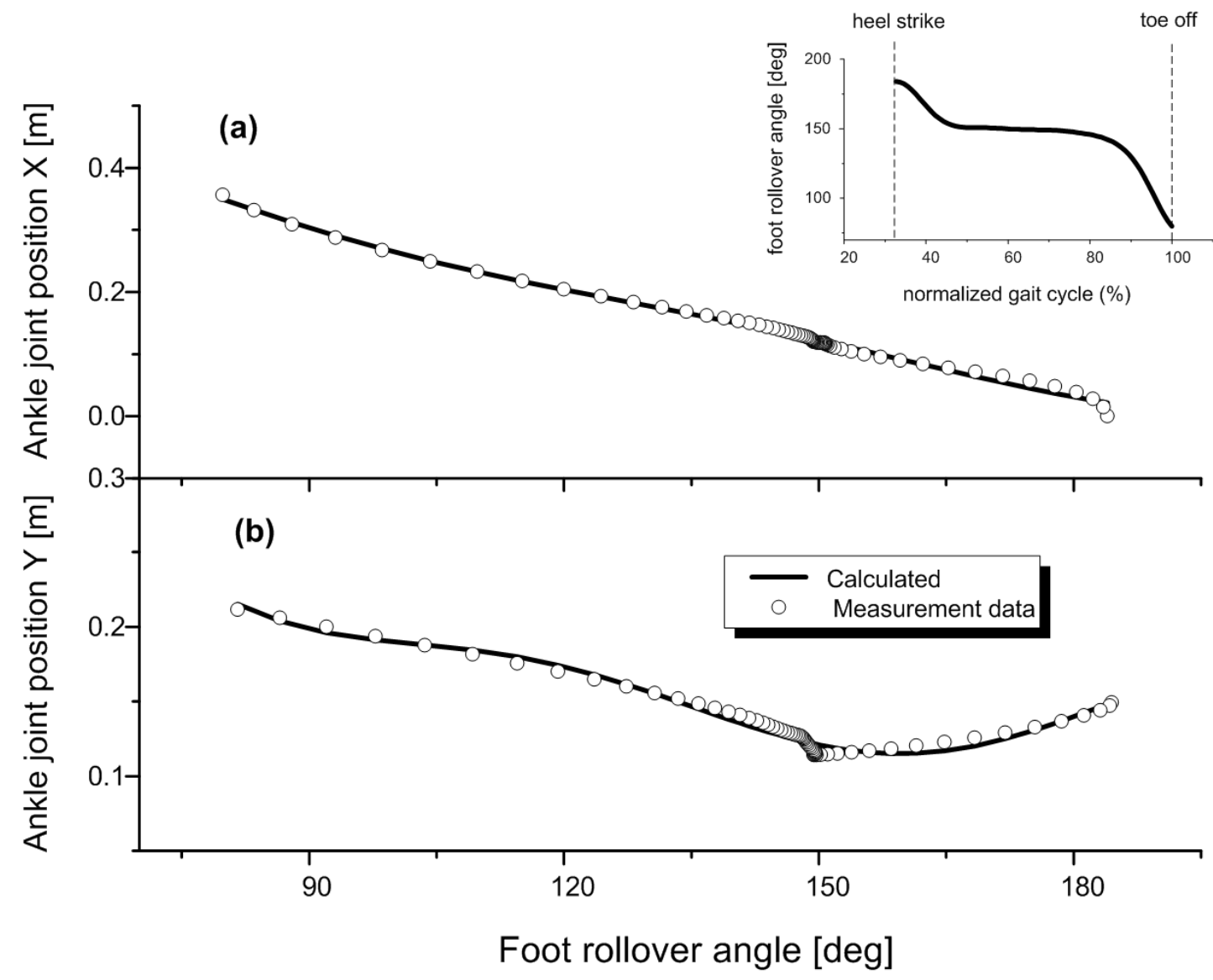

Figure 3 Mathematical representation of ankle-foot kinematics during stance phase, (a) $x$ coordinate of ankle joint and (b) $y$ coordinate of ankle joint, using $3^{\text {rd }}$ order Fourier series (black lines) compared with measurement data (circles). The subject (age: 38years, weight: $101.7 \mathrm{~kg}$, height: $178 \mathrm{~cm}$ ) walked at $1.52 \mathrm{~m} / \mathrm{s}$, and the cycle period was $0.98 \mathrm{~s}$. Inset is the time trajectory of stance foot rotation angle in the sagittal plane from heel strike to toe off, i.e. from $32 \%$ to $100 \%$ of the gait cycle. 


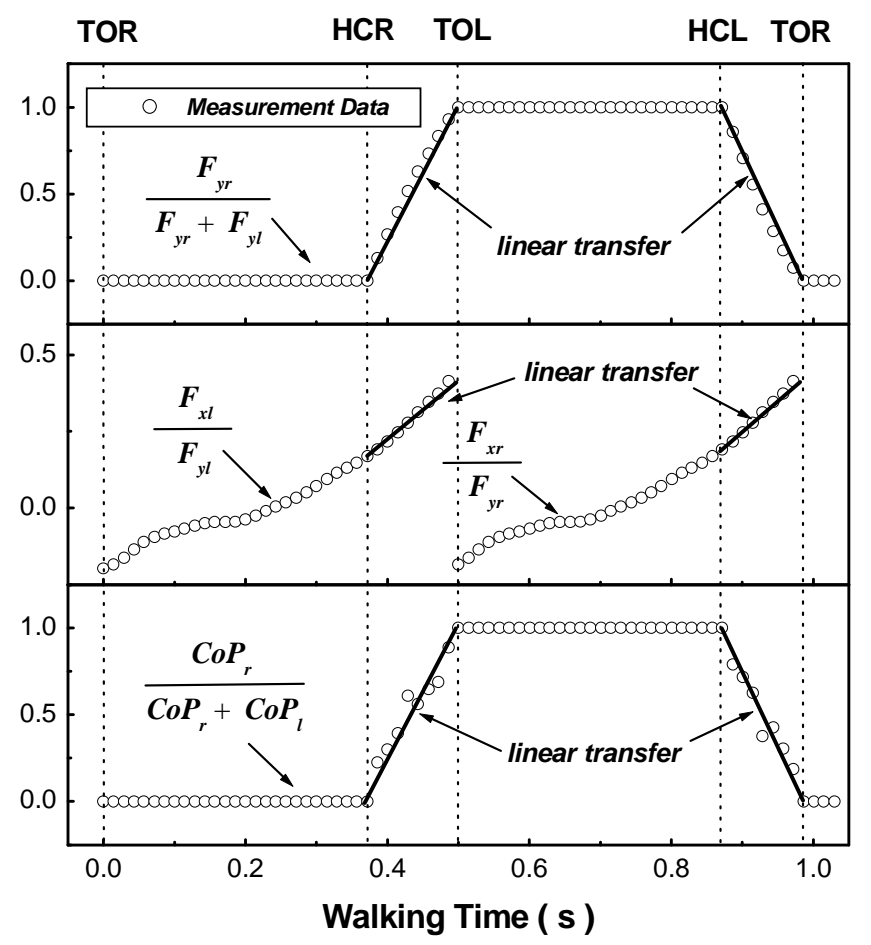

Figure 4 Calculated transfer ratios (solid line), based on linear assumptions, compared with measurement data from Winter (1990). $F_{x r}, F_{y r}, F_{x l}$ and $F_{y l}$ are the horizontal and vertical ground forces at the right and left foot. $\mathrm{CoP}_{r}$ and $\mathrm{CoP}_{l}$ are centres of pressure for right and left foot. $\mathrm{CoP}$ is defined as ground reaction moment about the ankle joint divided by vertical ground force $M_{z} / F_{y}$. In the double support phase from right heel contact (HCR) to left toe off (TOL), the vertical force transfer ratio $r_{t_{-} f y}$ increases from 0 to 1 , the horizontal force transfer ratio $r_{t_{-} f x}$ increases from $r_{t_{-} f x}^{(H C)}$ to $r_{t_{-} f x}^{(T O)}$, while the CoP transfer ratio $r_{t_{-} c o p}$ increases from 0 to 1 . 
(a) stiff-knee gait, energy cost: $510.50 \mathrm{~J}$

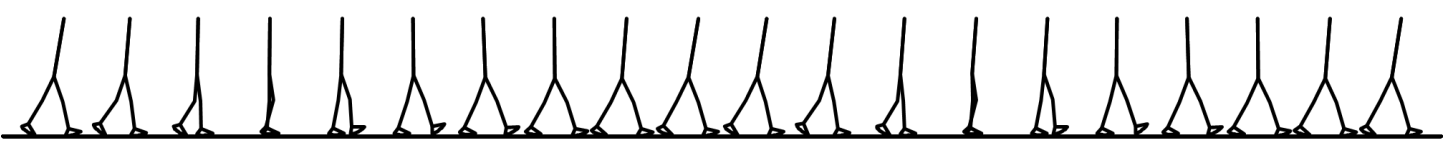

(b) inadequate knee extension, energy cost: $419.39 \mathrm{~J}$

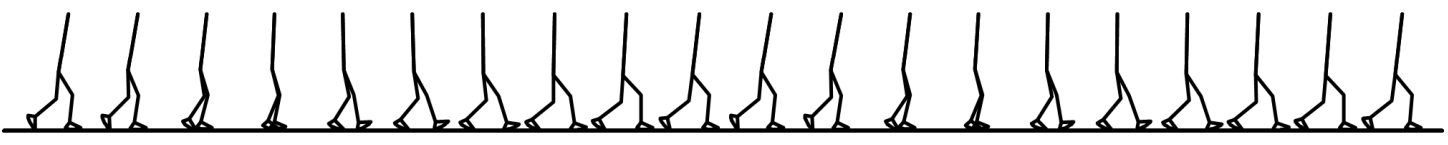

(c) excessive ankle plantar flexion, energy cost: $383.17 \mathrm{~J}$

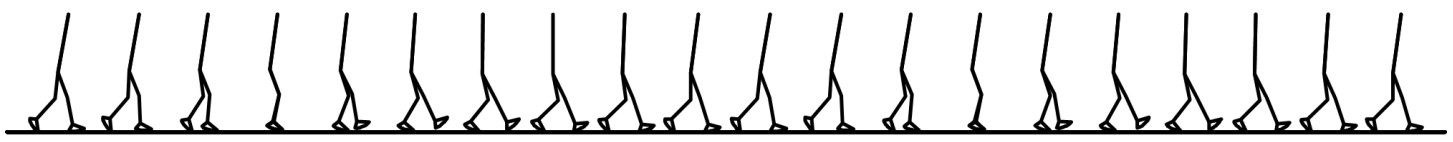

(d) predicted gait with lowest energy consumption, energy cost: $285.22 \mathrm{~J}$

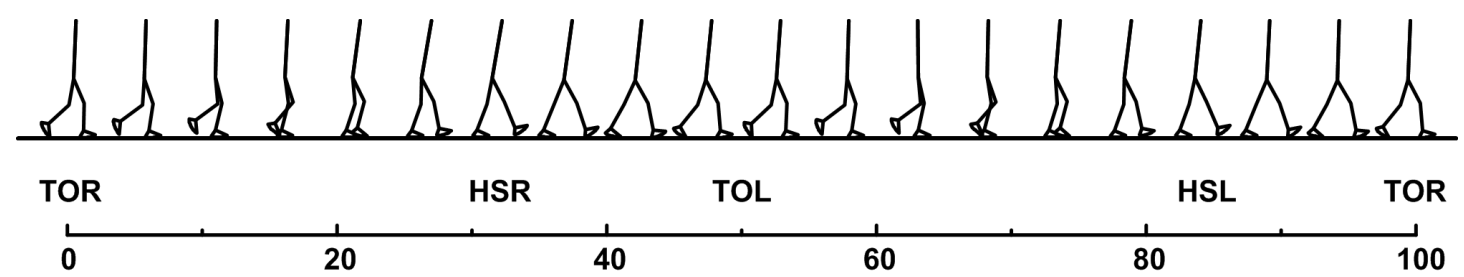

Figure 5 Some typical gait patterns (local minima) found during the random optimisation runs. The model (weight $101.7 \mathrm{~kg}$ ) walked at $1.50 \mathrm{~m} / \mathrm{s}$, with a cycle period of $1.0 \mathrm{~s}$. The right limb swing phase is from 0 to $32 \%$, and the stance phase is from $32 \%$ to $100 \%$. The double support phase is from 32 to $50 \%$ and from 82 to 100\%. (a) stiff-knee gait with limited knee flexion during swing phase, mechanical energy expenditure 510.50J. (b) inadequate knee extension in stance phase, energy cost 419.39J. (c) excessive ankle plantar flexion and consequently inadequate knee extension at opposite heel strike, energy cost 383.17J. (d) gait pattern which best reproduced natural human walking, lowest energy cost 285.22J. 

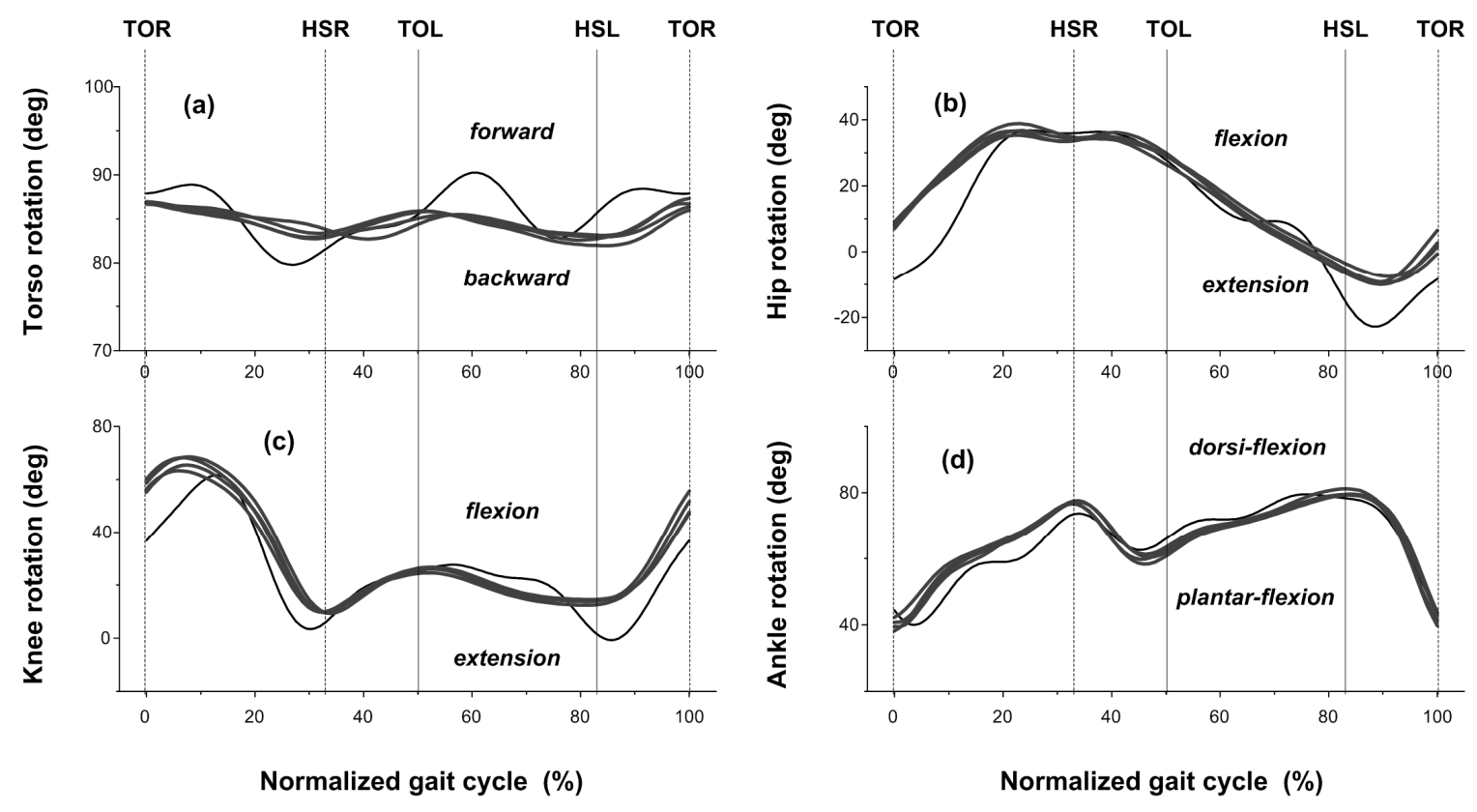

Figure 6 Predicted rotations of the trunk (a), right hip (b), right knee (c) and right ankle (d) in the sagittal plane (black lines), compared with measured data (grey lines) from 4 repeated trials for one subject (age: 38years, weight: $101.7 \mathrm{~kg}$, height: $178 \mathrm{~cm}$ ). The average walking speed was $1.50 \mathrm{~m} / \mathrm{s}$, and the average cycle period was $1.0 \mathrm{~s}$. The swing phase for the right limb is from 0 to $32 \%$, and the stance phase is from $32 \%$ to $100 \%$. The double support phase is from 32 to $50 \%$ and from 82 to $100 \%$. 


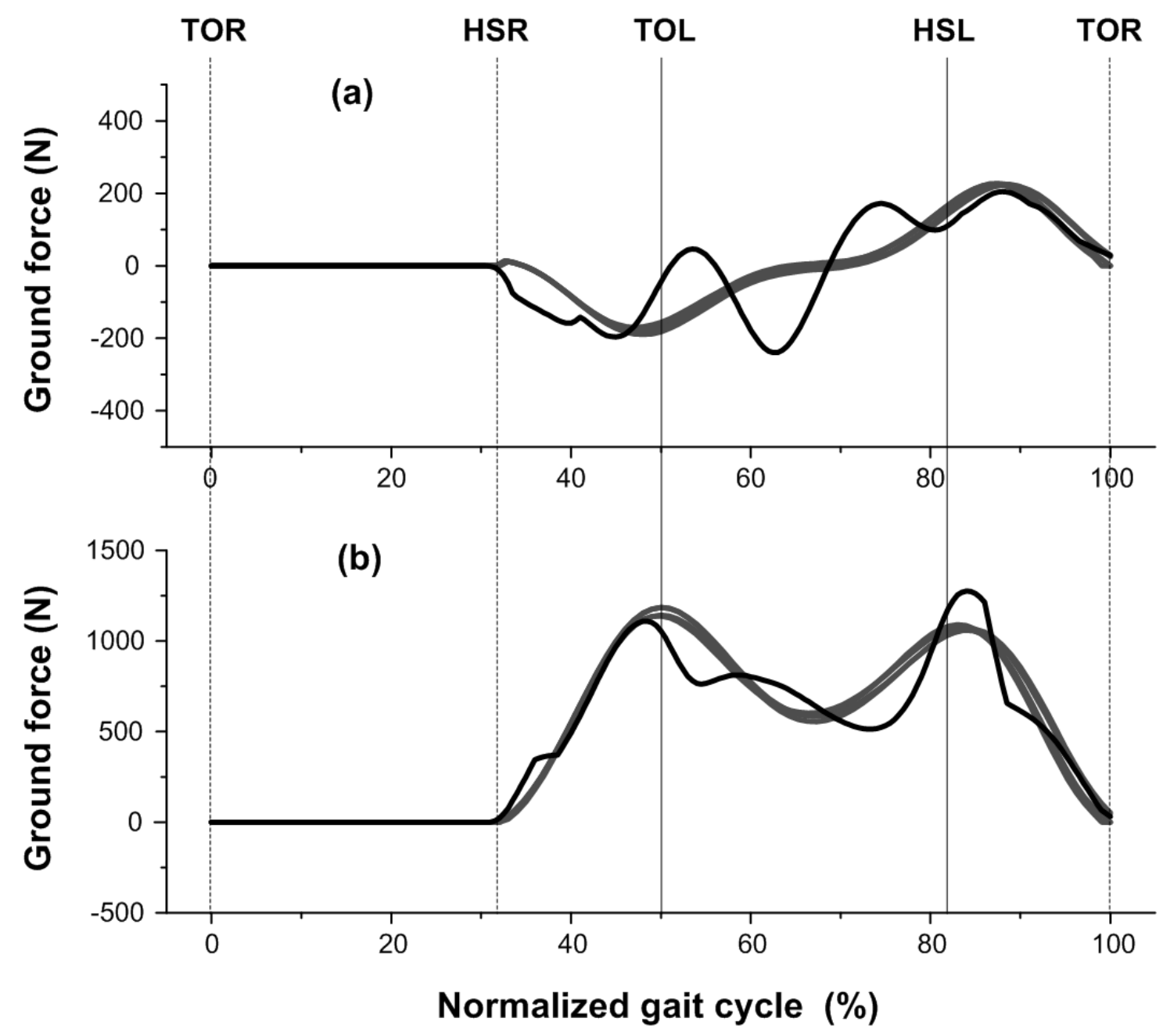

Figure 7 Predicted anterior-posterior ground reaction force (a) and vertical ground reaction force (b) (black lines), compared with recorded force plate data (grey lines) from 4 repeated trials for one subject (age: 38 years, weight: $101.7 \mathrm{~kg}$, height: $178 \mathrm{~cm}$ ). The average walking speed was $1.50 \mathrm{~m} / \mathrm{s}$, and the average cycle period was $1.0 \mathrm{~s}$. The swing phase for the right limb is from 0 to $32 \%$, and the stance phase is from $32 \%$ to $100 \%$. The double support phase is from 32 to $50 \%$ and from 82 to $100 \%$. 